\title{
Influence of Strain-Gage Balance Characteristics on the Precision Error of the Drag Coefficient
}

\author{
N. Ulbrich ${ }^{\dagger}$ \\ Jacobs Technology Inc., Moffett Field, California 94035 \\ A. L'Esperance $\ddagger$ \\ NASA Ames Research Center, Moffett Field, California 94035
}

\begin{abstract}
Improvements and results of a new method are presented that computes a pre-test estimate of the precision error of the drag coefficient of a wind tunnel model. The error estimate is defined as the part of the drag coefficient's precision error that is primarily associated with the precision error of the angle of attack measurement and physical characteristics of the chosen strain-gage balance. The method indirectly describes the precision error of the angle of attack measurement by using an assumed balance gage output variation of one microV/V. The physical characteristics of the balance, on the other hand, are described by partial derivatives of the axial and normal forces with respect to the strain-gage outputs. These derivatives can directly be obtained from the data reduction matrix of the balance. The precision error estimate itself is calculated by applying a simple explicit equation that uses the model reference area, the dynamic pressure, the angle of attack, the coefficients of the linear terms of the data reduction matrix, and the electrical output variation of one microV/ $\mathrm{V}$ as input. Precision errors at constant angle of attack may be visualized as contour plots by plotting them, for example, versus the Mach number and the total pressure. Characteristics of NASA's MC60E balance are used in combination with the reference area of a generic wind tunnel model in order to demonstrate that error estimates are independent of both the balance load format and the units chosen for the description of balance loads, model reference area, and the dynamic pressure. Finally, experimental data from a wind tunnel test of the Ames Check Standard Model in the NASA Ames 11-ft Transonic Wind Tunnel illustrates the application of the method to real-world test data.
\end{abstract}

\section{Nomenclature}

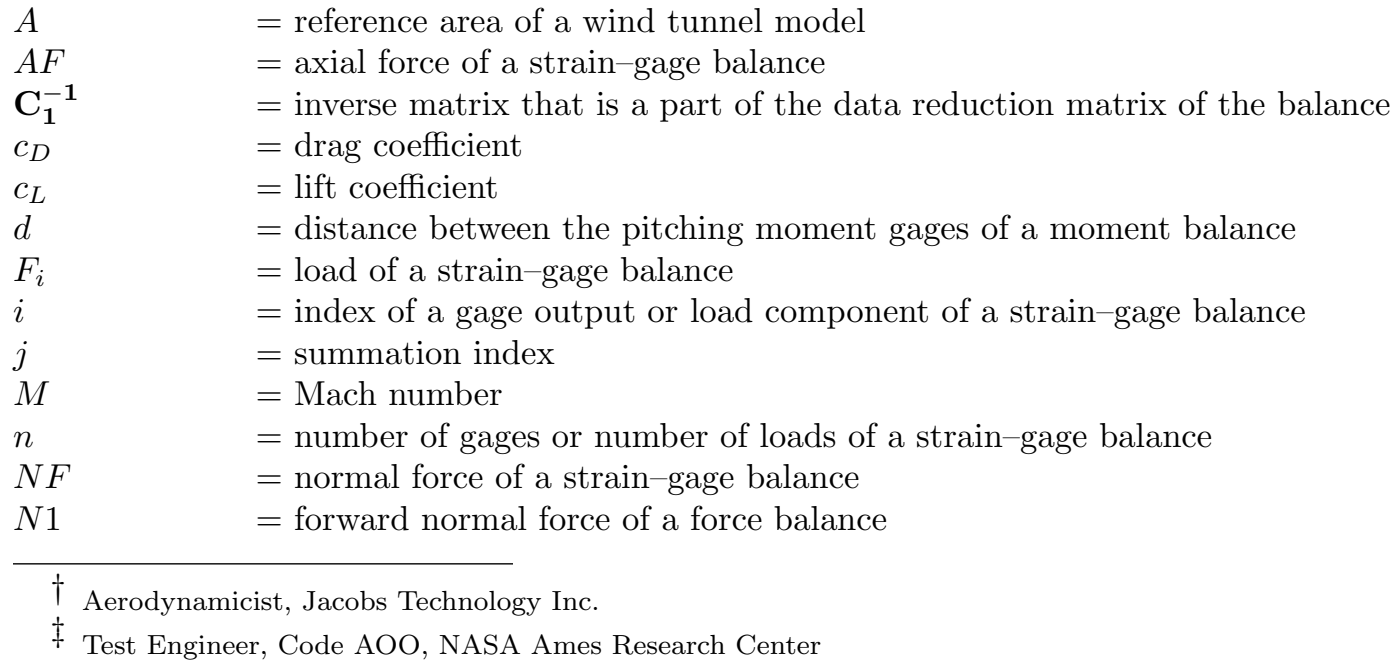




\begin{tabular}{|c|c|}
\hline N2 & $=$ aft normal force of a force balance \\
\hline$P M$ & $=$ pitching moment of a strain-gage balance \\
\hline$P M 1$ & $=$ forward pitching moment of a moment balance \\
\hline$P M 2$ & $=$ aft pitching moment of a moment balance \\
\hline$P_{T}$ & $=$ total pressure \\
\hline$Q$ & $=$ dynamic pressure \\
\hline$R e$ & $=$ Reynolds number \\
\hline$R_{i}$ & $=$ electrical output of a balance gage or bridge \\
\hline$S\left(F_{i}, R_{1}, \ldots\right)$ & $=$ upper bound of the partial derivatives of balance load component $F_{i}$ \\
\hline & $=$ angle of attack of a wind tunnel model \\
\hline & $=$ ratio of specific heats of a gas; ratio equals 1.4 for air \\
\hline$\left.\partial c_{D}\right|_{Q=\text { const }}$. & $=$ upper bound of the drag coefficient's precision error at constant dynamic pressu \\
\hline$\left.\delta c_{D}\right|_{\text {cou }}$ & $\begin{array}{l}=\text { upper bound of the drag coefficient's precision error in drag "counts" } \\
=\text { empirical upper bound of electrical output variations in units of }[\text { microV } / V]\end{array}$ \\
\hline
\end{tabular}

\section{Introduction}

The authors developed a new method in 2016 that computes a pre-test estimate of the upper bound of the precision error of the drag coefficient of a wind tunnel model so that the "repeatability" of the drag coefficient during a wind tunnel test can be assessed (for more detail see Ref. [1]). The upper bound is defined as a "conservative" estimate of the contribution of (i) the precision error of the angle of attack measurement and of (ii) physical characteristics of the strain-gage balance. Therefore, the method's analysis results may be used to compare the expected performance of different strain-gage balances that could be used for the drag force measurement during a wind tunnel test.

The authors' choice of focusing their attention on the contribution of (i) the precision error of the angle of attack measurement and of (ii) the balance characteristics to the precision error of the drag coefficient is supported by three conclusions. $\Rightarrow$ Conclusion 1: The contribution of the uncertainty of the dynamic pressure measurement is less that one drag "count" for most of the dynamic pressure range of a typical subsonic/transonic production wind tunnel (see Ref. [1], p. 6). $\Rightarrow$ Conclusion 2: The authors' method examines drag coefficient differences between "repeat" points; consequently, the precision error contribution associated with the use of a multivariate regression model of the balance calibration data for the load prediction can be neglected (the contribution will practically be identical for pairs of repeat points assuming that identical sets of electrical outputs are used for the prediction of the balance loads). $\Rightarrow$ Conclusion 3: Drag coefficient differences between sets of repeat points must primarily be associated with the precision error of the angle of attack measurement; this error can indirectly be "observed" as small differences between long-term averages of corresponding balance gage outputs of sets of repeat points; the authors relate this contribution to an empirical gage output variation of one microV/V (for more detail see Ref. [1], pp. 7-10).

It is assumed that balance loads are predicted during the wind tunnel test by using the Iterative Method. Therefore, a "data reduction matrix" must exist that describes the required load iteration scheme. This iteration scheme is used to predict balance loads from the measured electrical outputs of the balances gages (for more see Ref. [2], pp. 18-19). The authors' method was intentionally designed to use information about the balance characteristics that is contained in the coefficients of the data reduction matrix. Consequently, the authors' precision error estimate is given by an explicit equation that is only a function of (i) the data reduction matrix coefficients, (ii) the angle of attack, (iii) the model reference area, (iv) the dynamic pressure, and (v) an assumed balance gage output variation of one microV/V.

The authors' method can process data reduction matrices that are described in either direct-read, force balance, or moment balance format. Results of the precision error analysis are independent of the load format and the unit system that are used to describe loads, dynamic pressure, and model reference area. For convenience, computed precision error estimates are expressed in drag "counts" where one "count" equals 0.0001. Analysis results may be plotted versus Mach number and total pressure while keeping the angle of attack constant. Similarly, it is sometimes useful to plot analysis results versus Reynolds number and total pressure (see Ref. [1]). Recently, it was also decided to plot analysis results versus angle of attack and Mach number while keeping the total pressure constant. This plotting choice makes it easier to assess the expected 
precision error during an entire angle of attack sweep as the total pressure of a wind tunnel facility is usually kept constant during an angle of attack sweep. Table 1 below summarizes the plotting options that may be used to display the precision error of the drag coefficient.

Table 1: Plotting options for upper bound of the precision error of the drag coefficient.

\begin{tabular}{|c|c|c|c|}
\hline Option & Independent Variables & Constant Parameter & Comments \\
\hline \hline 1 & Mach number, total pressure & angle of attack & tunnel operator's choice \\
\hline 2 & Mach number, Reynolds number & angle of attack & customer's choice \\
\hline 3 & angle of attack, Mach number & total pressure & customer's choice \\
\hline
\end{tabular}

Basic details of the method to assess the precision error of the drag coefficient are provided in the next section of the paper. Afterwards, data from an assumed wind tunnel test are used to illustrate the application of the method. Finally, experimental data from a recent wind tunnel test of the Ames Check Standard Model in the NASA Ames 11-ft Transonic Wind Tunnel is used to compare the magnitude of predicted and observed precision errors of the drag coefficient.

\section{Precision Error Assessment}

The derivation of a pre-test estimate of the precision error of the drag coefficient of a wind tunnel model is discussed in detail in Ref. [1]. Therefore, only the most important assumptions related to definition and calculation of the precision error estimate are reviewed in this section. The reader must always keep in mind that the authors' method to obtain an estimate of the precision error of the drag coefficient does not assess the precision error of an isolated data point. Instead, it is examined how well drag coefficients of sets of repeat points are expected to agree. This type of comparison is typically used during a production wind tunnel test to quantify the overall quality of the drag coefficient measurement.

In general, the precision error of the drag coefficient of a wind tunnel model, i.e., the coefficient difference between sets of repeat data points, depends on measurement and/or prediction uncertainties of three main inputs that are used for the calculation of the drag coefficient. These three inputs are:

(i) dynamic pressure measurement,

(ii) balance load prediction,

(iii) angle of attack measurement.

The contribution of each one the three inputs above to the precision error of the drag coefficient needs to be discussed in more detail. First, the contribution associated with the uncertainty of the dynamic pressure measurement is examined. Fortunately, this contribution is small in a typical subsonic/transonic production wind tunnel when compared with the contributions of the balance load prediction and the angle of attack measurement. It can often be neglected as it only contributes on the order of one drag count (or less) to the total precision error of the drag coefficient for most of the operating envelope of a typical production wind tunnel (for a more detail see Ref. [1], pp. 5-6).

In the next step, the precision error contribution of the balance load prediction to the drag coefficient, i.e., the error contribution of the axial \& normal force prediction, is investigated in more detail. This task is complicated by the fact that the long-term average of the electrical output of a balance gage contains a part that is associated with the error of the "physical" angle of attack measurement. The unwanted part must be omitted so that the error contribution of the balance load prediction can clearly be separated from the error contribution of the angle of attack measurement. This problem can be addressed if the precision error contribution of the balance load prediction is assumed to be exclusively caused by load prediction imperfections associated with the use of the data reduction matrix of the balance. Furthermore, it is also assumed that (i) the balance temperature is constant during the wind tunnel test and that (ii) "observable" small differences between the measured outputs of sets of repeat points are exclusively caused by the error of the angle of attack measurement. Then, a part of the balance outputs of a set of repeat points can always be identified that (i) is identical for the set of repeat points and that (ii) is completely independent of the angle of attack measurement error. The authors use this specific part of the total outputs as input for the assessment of the precision error contribution of the balance load prediction. It was also mentioned above that the authors' method examines how well drag coefficients of sets of repeat points agree. In other 
words, coefficient differences between sets of repeat points are investigated. Consequently, the precision error contribution the balance load prediction can be neglected because (i) each repeat point is assumed to use the same part of the total outputs as input for the prediction of the balance loads and (ii) the resulting predicted balance loads of each repeat point are, by design, identical.

Finally, the precision error contribution of the angle of attack measurement needs to be examined. It is the main cause for "observable" differences between drag coefficients of sets of repeat points. It was mentioned in the previous paragraph that this precision error contribution can be described by small differences between long-term averages of corresponding electrical outputs of sets of repeat points. Then, the precision error contribution of the angle of attack measurement can be related to an empirical gage output variation of one microV/V (for more detail see Ref. [1], pp. 7-10). This approach is reasonable because (i) any "physical" angle of attack variation of the model in the wind tunnel flow field directly results in "physical" balance load variations, and, (ii) any "physical" balance load variation directly causes electrical output variations that can be observed at the axial and normal force gages of the balance. In other words, angle of attack variations and electrical output variations of the axial and normal force gages of the balance are directly coupled, i.e., they are not independent of each other. Therefore, electrical output variations can be used instead of angle of attack variations to describe the precision error contribution of the angle of attack measurement. Consequently, a precision error estimate of the drag coefficient can be defined that depends on an upper bound of the variations of the electrical outputs of the balance gages, the given balance characteristics, the model size, and the tunnel conditions.

Balance "characteristics" are hidden in the partial derivatives of the axial and normal force components of the balance assuming that each load component is derived with respect to each gage output. The derivatives can directly be obtained from the data reduction matrix coefficients of the balance. Then, it is possible to define an explicit equation of the precision error that is a function of (i) the balance characteristics, (ii) the angle of attack, (iii) the dynamic pressure, (iv) the model reference area, and (v) an empirical upper bound of the output variations of the axial and normal force gages of the balance. For clarity, the upper bound of the precision error is expressed in "counts" where one count equals 0.0001. Then, the following relationship for the precision error of the drag coefficient can be defined (see Ref. [1], Eqs. (15), (18)) ...

\section{UPPER BOUND OF PRECISION ERROR OF THE DRAG COEFFICIENT}

$$
\begin{gathered}
\left.\delta c_{D}\right|_{\text {counts }}=\left\{\left.10^{4} \cdot \delta c_{D}\right|_{Q=\text { const. }}\right\} \Longleftrightarrow \text { "counts" } \\
\text { where } \\
\left.\delta c_{D}\right|_{Q=\text { const. }} \leq|\varphi| \cdot \frac{S\left(A F, R_{1}, \ldots, R_{n}\right) \cdot \cos |\alpha|+S\left(N F, R_{1}, \ldots, R_{n}\right) \cdot \sin |\alpha|}{Q \cdot A}
\end{gathered}
$$

where $\varphi$ is an empirical upper bound of the electrical output variations of the gages, $\alpha$ is the angle of attack of the wind tunnel model, $Q$ is the dynamic pressure, $A$ is the reference area of the wind tunnel model, and $S(A F, \ldots)$ and $S(N F, \ldots)$ are a subset of the data reduction matrix coefficients that describe fixed characteristics of the relationship between predicted balance loads and measured electrical outputs of the balance gages. Each parameter on the right-hand side Eq. (1b) above has a useful interpretation that goes beyond its original definition. They can be summarized as follows:

$$
\begin{aligned}
& \varphi \equiv \text { upper bound of empirical output variations } \Longrightarrow \text { angle measurement error } \\
& S(A F, \ldots), S(N F, \ldots) \equiv \text { subset of matrix coefficients } \Longrightarrow \text { balance characteristics } \\
& \alpha \equiv \text { angle of attack } \Longrightarrow \text { model orientation relative to the free-stream direction } \\
& Q \equiv \text { dynamic pressure } \Longrightarrow \text { tunnel conditions that the model experiences } \\
& A \equiv \text { model reference area } \Longrightarrow \text { parameter that describes the model size }
\end{aligned}
$$

American Institute of Aeronautics and Astronautics 
These interpretations make it clear that a good compromise between the chosen (i) balance characteristics, (ii) tunnel conditions, and (iii) model size has to be found during preparation and execution of a wind tunnel test in order to minimize the precision error of the drag coefficient. This goal is not easily achieved as the characteristics of the chosen balance, i.e., the built-in sensitivities of the axial and normal force gages, are dictated by the maximum dynamic pressure that the wind tunnel model can experience.

Past experience has shown that the value of one microV/V is a suitable choice for the upper bound $\varphi$ of the output variations. This value describes the angle of attack measurement error by using a single "global" value. The magnitude of the upper bound was confirmed after reviewing repeat data from (i) wind tunnel tests at the NASA Ames 11-ft Transonic Wind Tunnel and (ii) strain-gage balance calibrations. It is possible to interpret the choice of one microV/V in a practical context if the relationship between output and angle of attack change is assumed to be linear. Then, one microV/V corresponds to an angle measurement error of $0.01[\mathrm{deg}]$ if the axial \& normal force gages of the balance have a maximum output of $1000 \mathrm{microV} / \mathrm{V}$ that occurs at an angle of attack of $10[\mathrm{deg}]$. This interpretation can be summarized as follows:

$$
|\varphi| \approx 1.0[\mathrm{microV} / \mathrm{V}] \equiv 0.01[\mathrm{deg}] \times \frac{1000[\mathrm{microV} / \mathrm{V}]}{10[\mathrm{deg}]}
$$

The strain-gage balance characteristics themselves are "hidden" in the terms $S\left(A F, R_{1}, \ldots, R_{n}\right)$ and $S\left(N F, R_{1}, \ldots, R_{n}\right)$ that are used in Eq. (1b) above. They represent the upper bounds of the partial derivatives of each load component with respect to the gage outputs of the given $n$-component balance. In general, this upper bound of the partial derivatives is defined in Ref. [1] as follows ...

$$
S\left(F_{i}, R_{1}, \ldots, R_{n}\right) \leq \sqrt{\left[\frac{\partial F_{i}}{\partial R_{1}}\right]^{2}+\cdots+\left[\frac{\partial F_{i}}{\partial R_{n}}\right]^{2}}=\sqrt{\sum_{j=1}^{n}\left[\frac{\partial F_{i}}{\partial R_{j}}\right]^{2}}
$$

where $F_{i}$ is the symbol of the $i$-th load component of the balance. Then, after setting the generic load symbol $F_{i}$ to be the axial force component $A F$ of the balance, the upper bound of the partial derivatives of the axial force component of the balance is defined by the following equation:

$$
S\left(A F, R_{1}, \ldots\right) \leq \sqrt{\sum_{j=1}^{n}\left[\frac{\partial A F}{\partial R_{j}}\right]^{2}}
$$

Similarly, the upper bound of the partial derivatives of the normal force can be written as follows assuming that balance loads are given in direct-read format $(A F, N F, P M, \ldots)$ :

$$
S\left(N F, R_{1}, \ldots\right) \leq \sqrt{\sum_{j=1}^{n}\left[\frac{\partial N F}{\partial R_{j}}\right]^{2}}
$$

Unfortunately, the calculation of the upper bound of the partial derivatives of the normal force of the balance is more complicated because balance loads can also be expressed in force balance format $(A F, N 1, N 2, \ldots)$ or moment balance format $(A F, P M 1, P M 2, \ldots)$. Therefore, in order to address this issue, the authors initially applied approximations of the upper bound of the partial derivatives of the normal force whenever balance data was given in force balance or moment balance format (see Ref. [1], Eqs. (16a), 16b). These approximations, however, were recently replaced by exact expressions so that the upper bound computed in either force balance or moment balance format would match the value that is obtained if balance loads are processed in direct-read format. This important improvement of the authors' original method makes the precision error estimate of the drag coefficient independent of the chosen balance load format.

The newly developed exact expression for the upper bounds of the partial derivatives of the normal force are derived in detail in the appendix of the paper. Therefore, only final results are listed below. First, the upper bound of the partial derivatives of the normal force is listed assuming that balance loads are given in force balance format $(A F, N 1, N 2, \ldots)$. Then, using Eq. (12) from the appendix of the paper, we get:

$$
S\left(N F, R_{1}, \ldots\right) \leq \sqrt{\sum_{j=1}^{n}\left[\frac{\partial N 1}{\partial R_{j}}\right]^{2}+\sum_{j=1}^{n}\left[\frac{\partial N 2}{\partial R_{j}}\right]^{2}+2 \cdot \sum_{j=1}^{n}\left[\frac{\partial N 1}{\partial R_{j}} \cdot \frac{\partial N 2}{\partial R_{j}}\right]}
$$


Similarly, assuming that the distance " $d$ " between the pitching moment gages of the moment balance is known, an upper bound of the partial derivatives of the normal force can be obtained if balance loads are given in moment balance format $(A F, P M 1, P M 2, \ldots)$. Then, using Eq. (16) from the appendix, we get:

$$
S\left(N F, R_{1}, \ldots\right) \leq \frac{1}{d} \cdot \sqrt{\sum_{j=1}^{n}\left[\frac{\partial P M 1}{\partial R_{j}}\right]^{2}+\sum_{j=1}^{n}\left[\frac{\partial P M 2}{\partial R_{j}}\right]^{2}-2 \cdot \sum_{j=1}^{n}\left[\frac{\partial P M 1}{\partial R_{j}} \cdot \frac{\partial P M 2}{\partial R_{j}}\right]}
$$

The partial derivatives $\partial A F / \partial R_{j}, \ldots, \partial P M 2 / \partial R_{j}$ used in Eqs. (4) to (5c) above are easily obtained. They can be found among the coefficients of the inverse matrix $\mathbf{C}_{\mathbf{1}}^{-\mathbf{1}}$ that is a part of the data reduction matrix of the balance for the given load format assuming the Iterative Method is used to predict balance loads from measured gage outputs (for more detail see Ref. [1], p. 15, or, Ref. [2], pp. 18-19).

The dynamic pressure in compressible flow is also needed in order to apply Eq. (1b) above. It depends on the "tunnel conditions" that are described by the ratio of specific heats of the gas $(\gamma)$, the total pressure $\left(P_{T}\right)$, and the Mach number $(M)$. Then, we get the following equation (taken from Ref. [3], Eq. (48)):

$$
Q=P_{T} \cdot \frac{\gamma}{2} \cdot M^{2} \cdot\left[1+\frac{\gamma-1}{2} \cdot M^{2}\right]^{-\gamma /(\gamma-1)}
$$

Then, knowing that the ratio $\gamma$ of specific heats of air equals 1.4, Eq. (6a) can also be written as follows:

$$
\text { air } \Longrightarrow \gamma=1.4 \Longrightarrow Q=P_{T} \cdot(7 / 10) \cdot M^{2} \cdot\left[1+0.2 \cdot M^{2}\right]^{-7 / 2}
$$

At this point, the upper bound of the drag coefficient's precision error can be computed as all quantities on the right-hand side of Eq. (1b) above are defined. An example of the calculation of a precision error estimate of the drag coefficient is discussed in the next section.

\section{Discussion of Example}

A hypothetical combination of a generic wind tunnel model of an airplane with a six-component straingage balance is used in this section to illustrate the calculation of a precision error of the drag coefficient. The analysis is done in both direct-read and force balance format to quantify the influence of the load format choice on the precision error. In addition, the precision error analysis in force balance format is also carried out in SI units so that the independence of the estimate from the chosen unit system can be shown.

The chosen generic wind tunnel model is to be tested in the NASA Ames 11-ft Transonic Wind Tunnel. The model has a reference area of $4.5[\mathrm{sqft}]$ or $0.4181[\mathrm{sqm}]$. It was also assumed that the model is fitted to NASA's MC60E six-component force balance. Table 2 below shows the nominal axial and normal force capacities of this balance for three selected balance load formats.

Table 2: Axial and normal force capacities of the MC60E balance (English Engineering and SI units).

\begin{tabular}{|c|c|c|c|c|c|}
\hline & Load Format & $A F$ & $N F$ & $N 1$ & $N 2$ \\
\hline \hline Case 1 & Direct-Read ${ }^{\dagger}$ & $700[l b f]$ & $5000[l b f](P M=0)$ & - & - \\
\hline Case 2 & Force Balance $^{\dagger}$ & $700[l b f]$ & - & $2500[l b f]$ & $2500[l b f]$ \\
\hline Case 3 & Force Balance ${ }^{\ddagger}$ & $3114[N]$ & - & $11121[N]$ & $11121[N]$ \\
\hline \multicolumn{7}{|c|}{$\dagger^{\dagger}$ loads described in English engineering units ; ${ }^{\ddagger}$ loads described in SI units }
\end{tabular}

Machine calibration data of the MC60E balance was analyzed for the three load formats. Afterwards, a data reduction matrix was generated for each case assuming that the Iterative Method is used for the balance load prediction. Then, the upper bound of the partial derivatives of the axial and normal force components was computed by using Eqs. (4), (5a), and (5b) and the applicable coefficients of inverse matrix $\mathbf{C}_{\mathbf{1}}^{-\mathbf{1}}$ from the data reduction matrix (for more details see Ref. [1], p. 15, or, Ref. [2], pp. 18-19). Table 3 below shows the numerical values of the computed upper bounds of the partial derivatives. 
Table 3: Upper bound of the partial derivatives of the axial and normal force of the MC60E balance.

\begin{tabular}{|c|c|c|c|c|}
\hline & Load Format & $S\left(A F, R_{1}, \ldots, R_{n}\right)$ & $S\left(N F, R_{1}, \ldots, R_{n}\right)$ & Comments \\
\hline Case 1 & $\begin{array}{l}\text { Direct-Read }{ }^{\dagger} \\
\text { (English units) }\end{array}$ & $\begin{array}{c}0.8548 \\
{[l b f /(\text { microV } / V)]}\end{array}$ & $\begin{array}{c}3.2131 \\
{[l b f /(\text { microV } / V)]}\end{array}$ & $\begin{array}{l}S(A F, \ldots) \Longrightarrow \text { Eq. (4) } \\
S(N F, \ldots) \Longrightarrow \text { Eq. (5a) }\end{array}$ \\
\hline Case 2 & $\begin{array}{l}\text { Force Balance }{ }^{\dagger} \\
\text { (English units) }\end{array}$ & $\begin{array}{c}0.8548 \\
{[l b f /(\text { microV } / V)]}\end{array}$ & $\begin{array}{c}3.2131 \\
{[l b f /(\text { microV } / V)]}\end{array}$ & $\begin{array}{l}S(A F, \ldots) \Longrightarrow \text { Eq. }(4) \\
S(N F, \ldots) \Longrightarrow \text { Eq. }(5 b)\end{array}$ \\
\hline Case 3 & $\begin{array}{l}\text { Force Balance } \\
\quad \text { (SI units) }\end{array}$ & $\begin{array}{c}3.8023 \\
{[N /(\text { microV } / V)]}\end{array}$ & $\begin{array}{c}14.2925 \\
{[N /(\text { microV } / V)]}\end{array}$ & $\begin{array}{l}S(A F, \ldots) \Longrightarrow \text { Eq. }(4) \\
S(N F, \ldots) \Longrightarrow \text { Eq. }(5 b)\end{array}$ \\
\hline
\end{tabular}

Results shown in Table 3 above illustrate the fact that the values of $S(A F, \ldots)$ and $S(N F, \ldots)$ of Case 1 match corresponding values that are reported for Case 2 even though (i) the balance loads were expressed using different load formats and (ii) completely different sets of partial derivatives were used to compute the upper bounds. This observation confirms that the constants $S(A F, \ldots)$ and $S(N F, \ldots)$ represent physical balance "characteristics" that are independent of the chosen balance load format. Another observation can be made in Table 3 after comparing the upper bounds of Case 2 with the upper bounds of Case 3 . The ratio between $S(A F, \ldots)$ and $S(N F, \ldots)$ is, by definition, a dimensionless quantity. Therefore, the ratio must be independent of the chosen units. Consequently, the ratio obtained from the numerical values of Case 2 equals the ratio that is obtained from the numerical values of Case 3 (i.e., $0.8548 / 3.2131=3.8023 / 14.2925$ ).

Precision errors of the drag coefficient for Case 1 of Tables $2 \& 3$ were computed in the next step by applying Eqs. $(1 a)$ and $(1 b)$ and assuming that the balance loads are given in direct-read format. The required dynamic pressure for each combination of Mach number and total pressure is shown in Fig. 1 below.

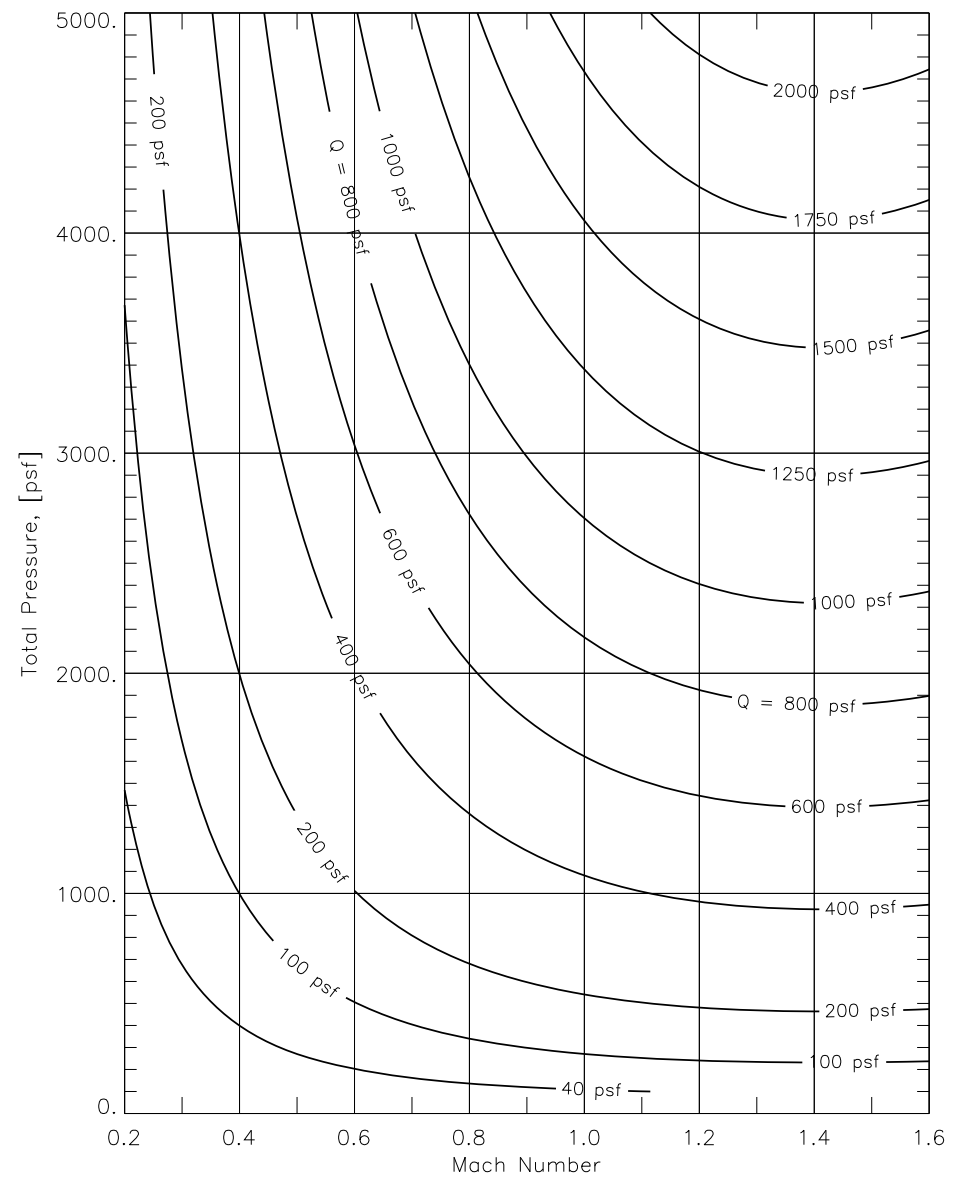

Fig. 1 Dynamic pressure versus Mach number and total pressure $(\gamma=1.4$, pressure unit $\equiv[\mathrm{psf}])$. 
Corresponding values were used in Eq. (1b) as input. Figure 2a below shows the resulting precision error of the drag coefficient in "counts" plotted versus Mach number and total pressure (one "count" corresponds to 0.0001 ). These precision error estimates were computed by keeping the angle of attack constant at $5^{\circ}$.

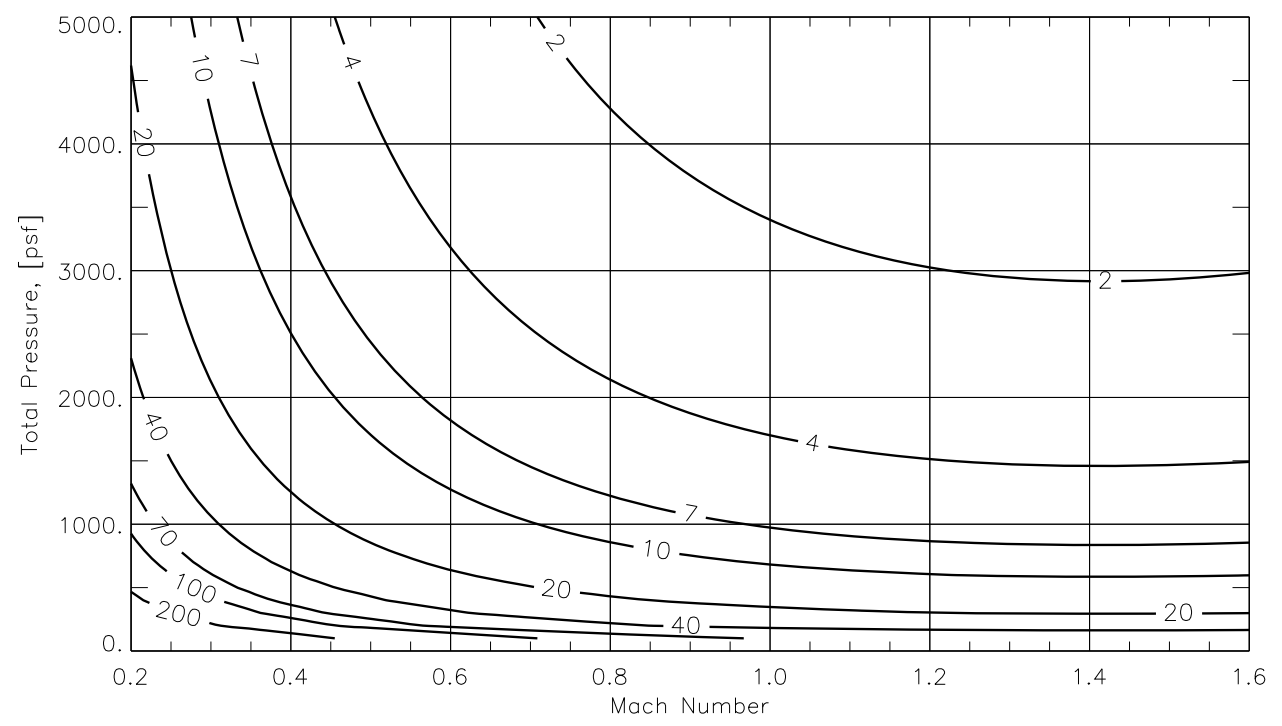

Fig. 2a Case 1: Precision error of the drag coefficient plotted in "counts" versus Mach number and total pressure ( $\underline{\text { direct-read format }}$, one "count" $=0.0001$, angle of attack $=$ const. $=5^{\circ}$ ).

Figure $2 \mathrm{~b}$ below shows the resulting precision error of the drag coefficient when plotted versus angle of attack and Mach number. This time, the total pressure was fixed at 2116.213 [psf].

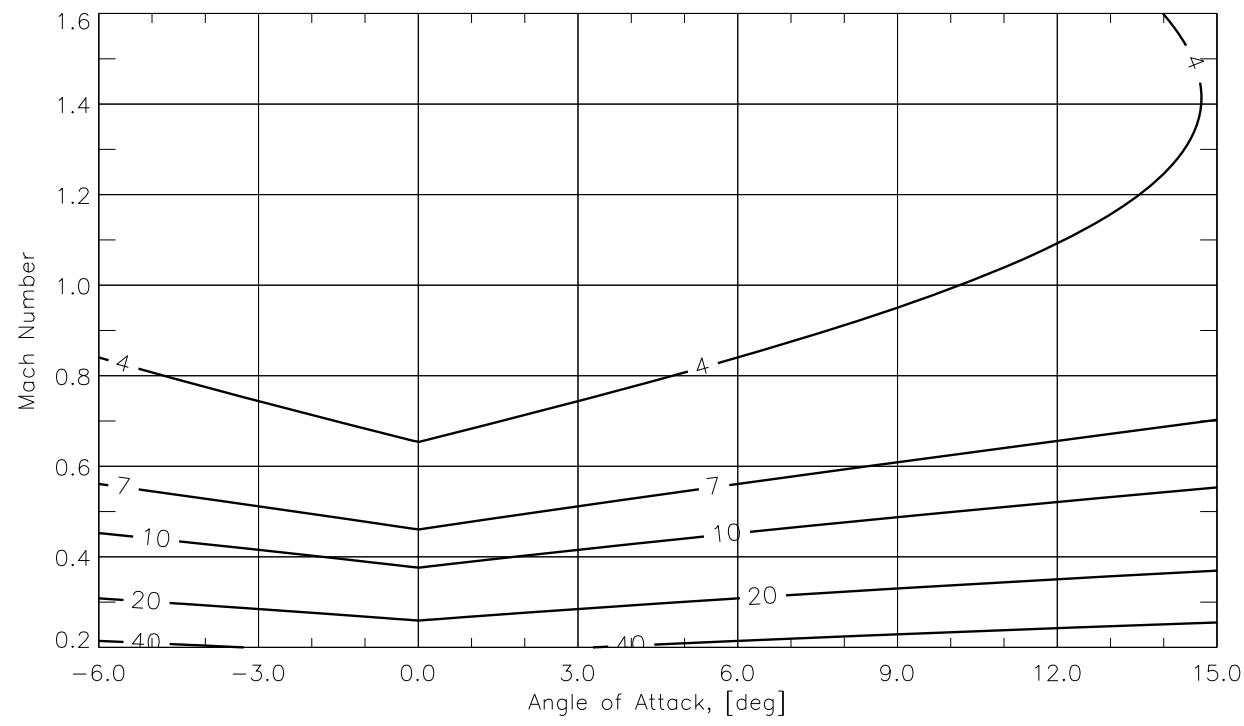

Fig. 2b Case 1: Precision error of the drag coefficient plotted in "counts" versus angle of attack and Mach number $(\underline{\text { direct-read format }}$, one "count" $=0.0001$, total pressure $=$ const. $=2116.213[\mathrm{psf}]$ ).

Then, precision errors of the drag coefficient for Case 2 of Table $2 \& 3$ were computed assuming that balance loads are given in force balance format. Figure 3 a below shows the resulting precision error of the 
drag coefficient when plotted versus Mach number and total pressure.

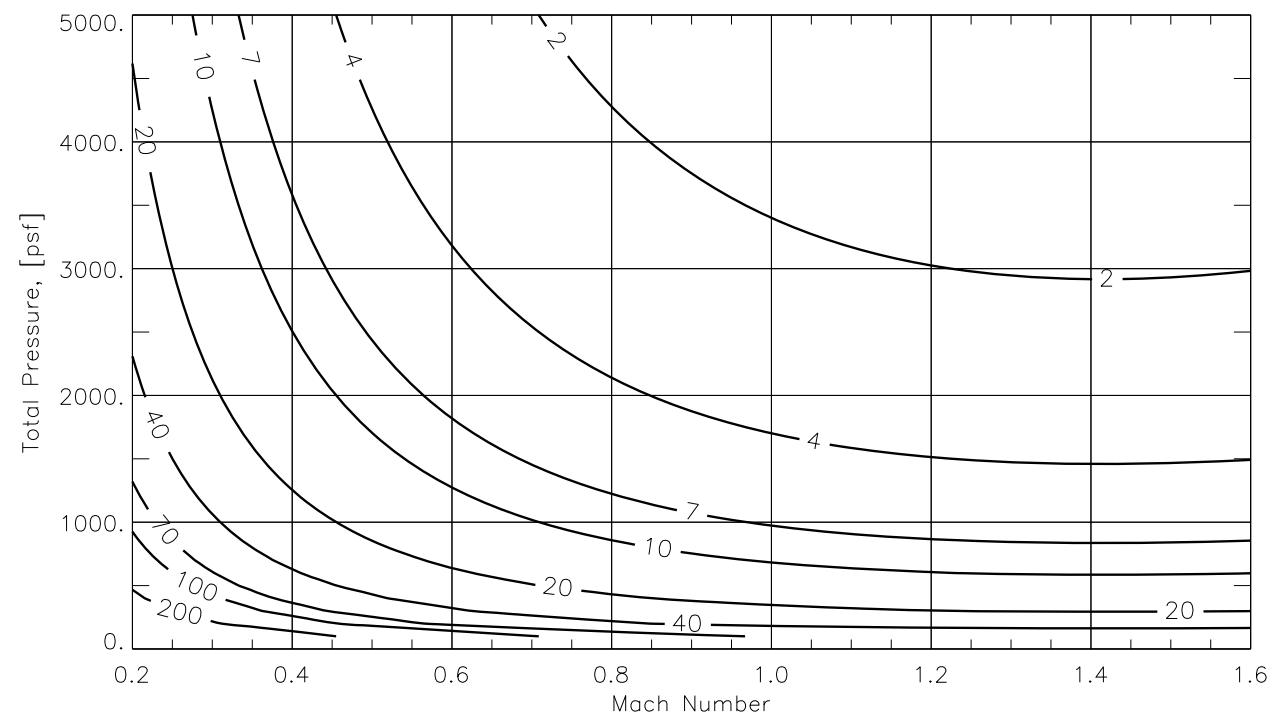

Fig. 3a Case 2: Precision error of the drag coefficient plotted in "counts" versus Mach number and total pressure ( force balance format, one "count" $=0.0001$, angle of attack $=$ const. $=5^{\circ}$ ).

Similarly, Figure $3 \mathrm{~b}$ below shows the resulting precision error of the drag coefficient when plotted versus angle of attack and Mach number.

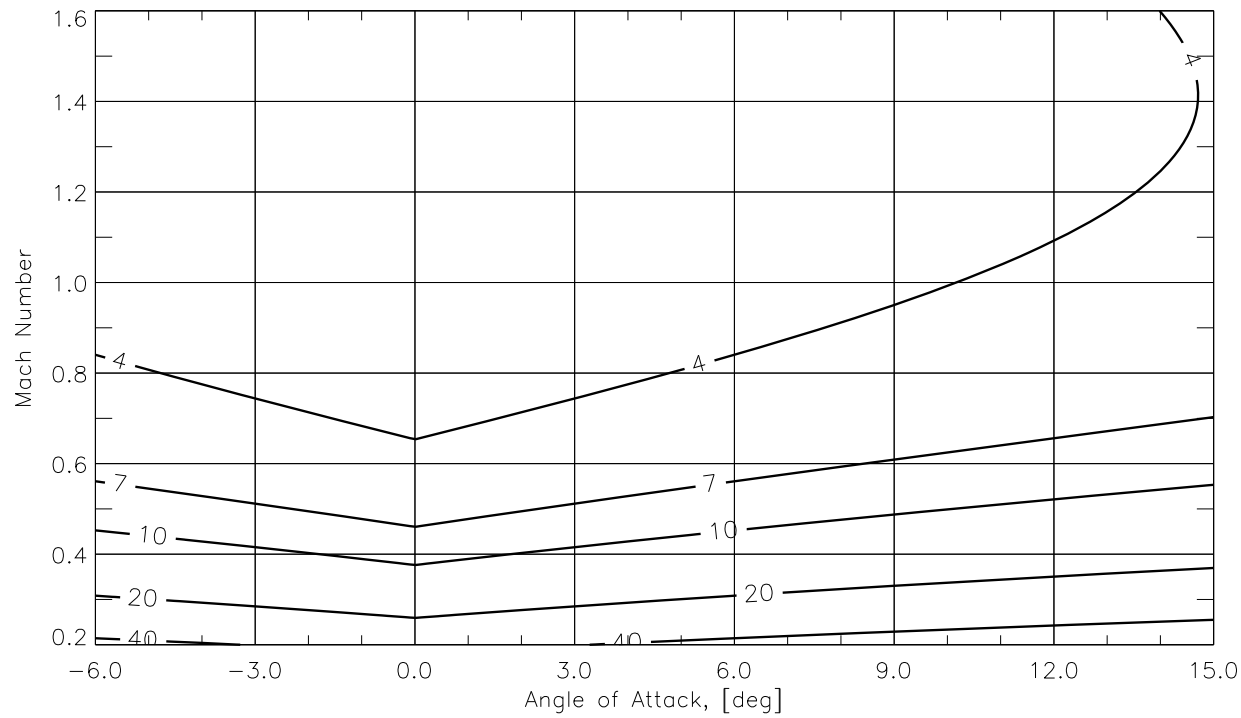

Fig. 3b Case 2: Precision error of the drag coefficient plotted in "counts" versus angle of attack and Mach number ( $\underline{\text { force balance format }}$, one "count" $=0.0001$, total pressure $=$ const. $=2116.213[\mathrm{psf}]$ ).

As expected, precision error estimates shown in Figs. 2a \& 2b for direct-read format exactly match corresponding values that are shown in Figs. 3a \& 3b for force balance format. This observation confirms an important characteristics of the authors' method: the precision error estimates of the drag coefficient are independent of the balance load format. Finally, precision errors of the drag coefficient for Case 3 in Table $2 \& 3$ were computed assuming that (i) balance loads are given in force balance format and (ii) SI units are used. The required dynamic pressure in SI units for each combination of Mach number and total pressure is shown in Fig. 4 below.

American Institute of Aeronautics and Astronautics 


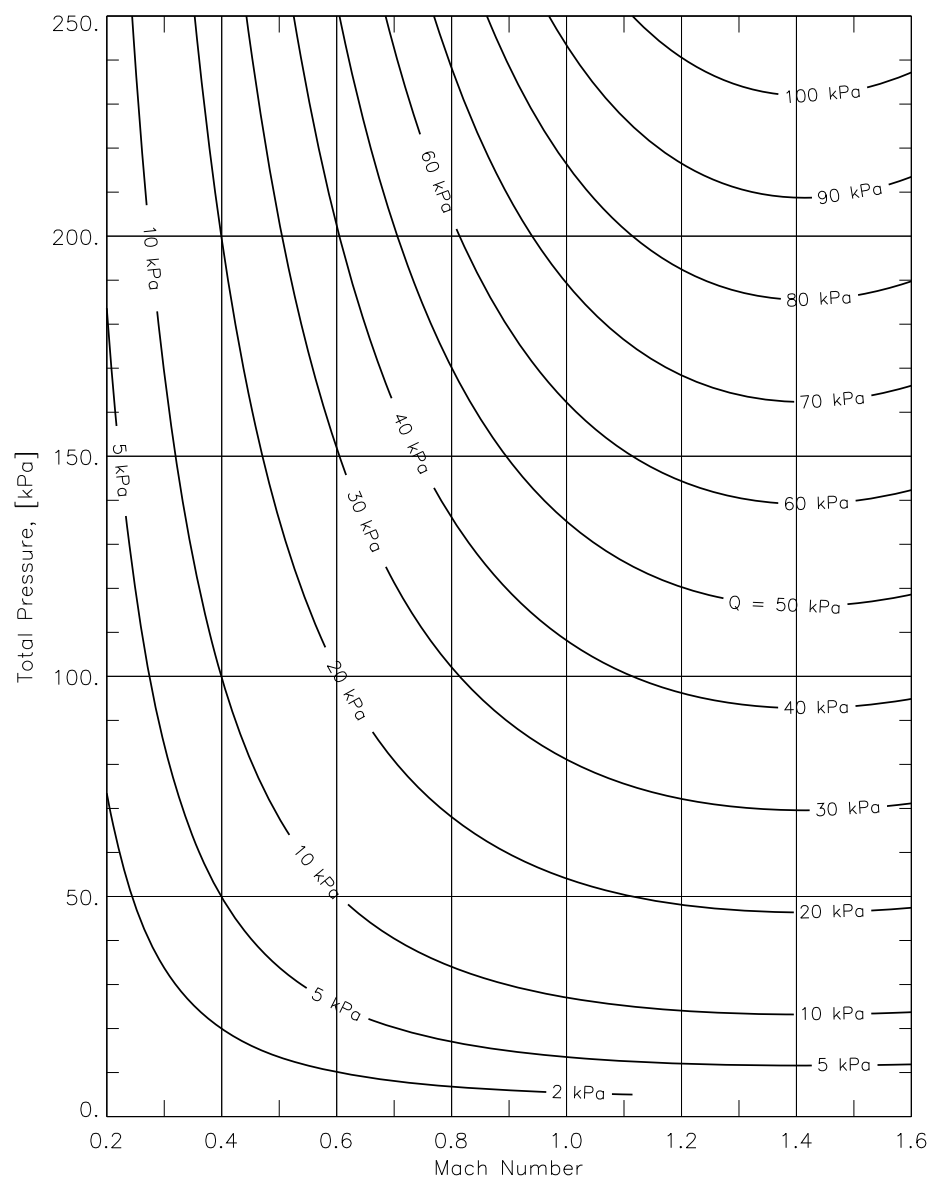

Fig. 4 Dynamic pressure plotted versus Mach number and total pressure $(\gamma=1.4$, pressure unit $\equiv[\mathrm{kPa}])$.

Figure 5a below shows the resulting precision error of the drag coefficient plotted versus Mach number and total pressure. These error estimates were computed by keeping the angle of attack constant at $5^{\circ}$.

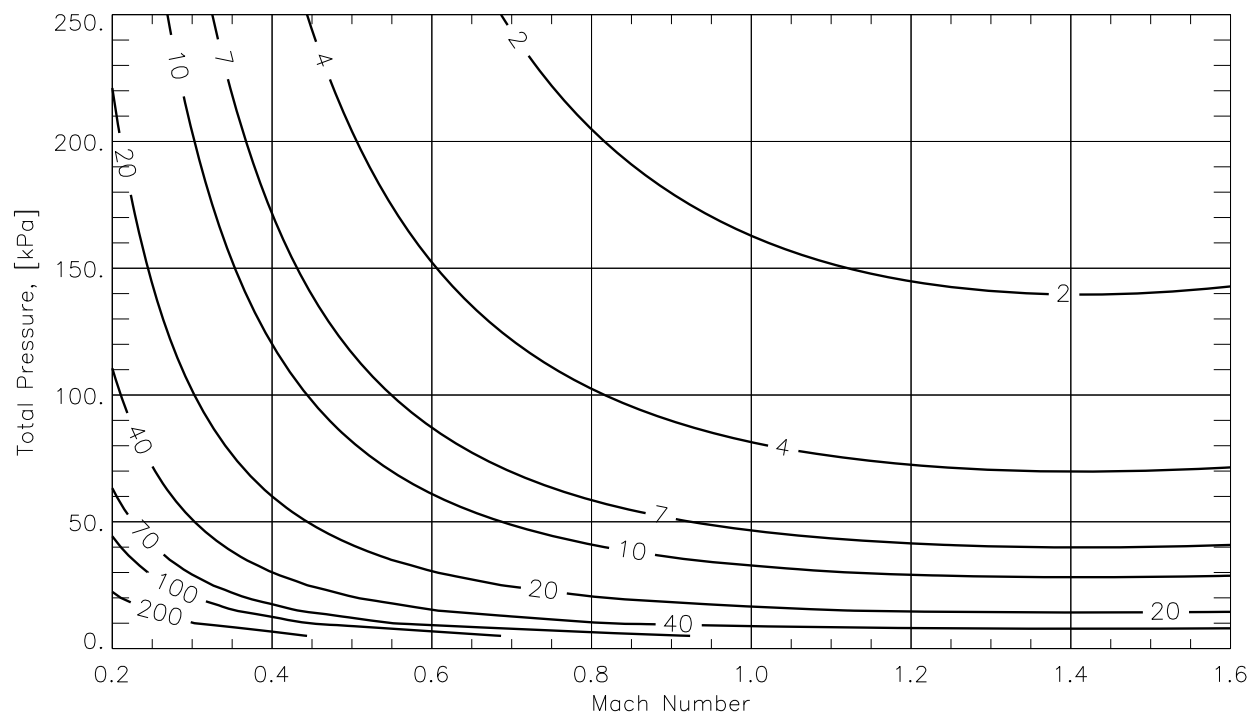

Fig. 5a Case 3: Precision error of the drag coefficient plotted in "counts" versus Mach number and total pressure ( $\underline{\text { force balance format }}, \underline{\text { SI units, }}$, one "count" $=0.0001$, angle of attack $=$ const. $=5^{\circ}$ ). 
Figure 5b below shows the resulting precision error of the drag coefficient when plotted versus angle of attack and Mach number. This time, the total pressure was fixed at $101.325[\mathrm{kPa}]$.

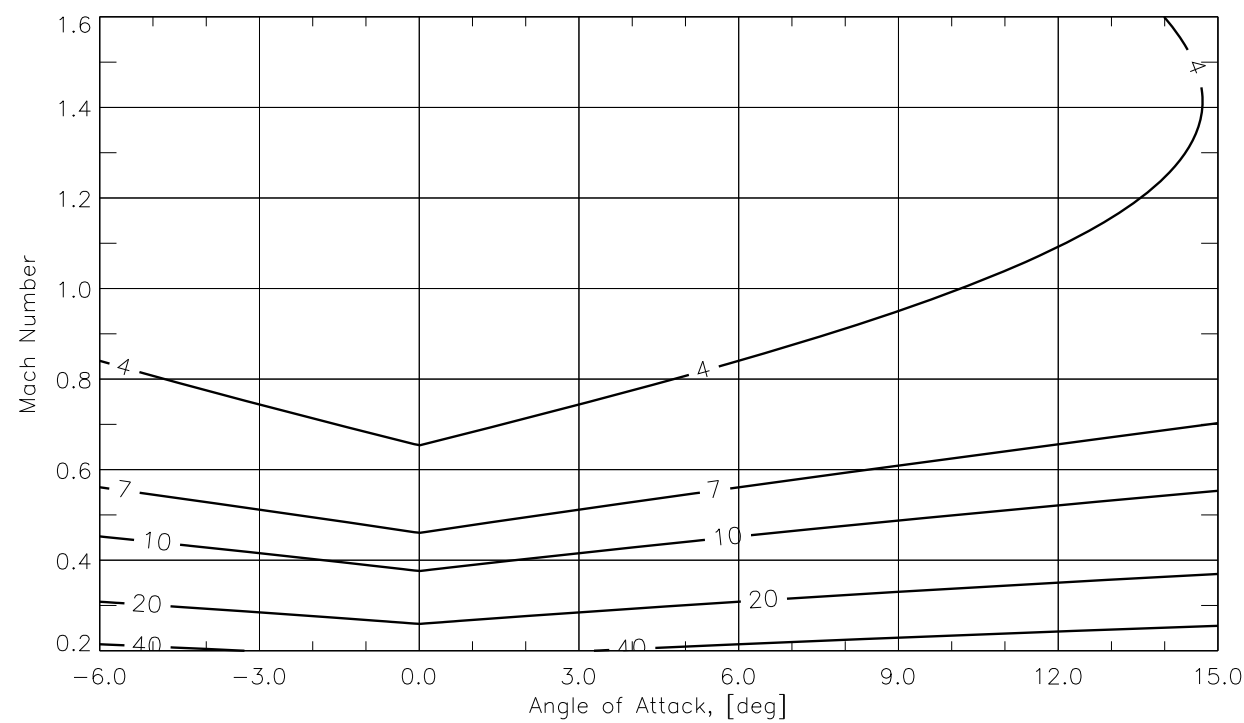

Fig. 5b Case 3: Precision error of the drag coefficient plotted in "counts" versus angle of attack and Mach number; ( force balance format, , one "count" $=0.0001$, total pressure $=$ const. $=101.325[\mathrm{kPa}]$ ).

No differences between corresponding precision errors estimates are observed when results depicted in Fig. 3b (English engineering units) are compared with corresponding results depicted in Fig. 5b (SI units). This observation is expected as the precision error estimates must be independent of the unit system that is used for analysis. - Data from a test of the Ames Check Standard Model in the NASA Ames 11-ft Transonic Wind Tunnel is analyzed in the next section to illustrate the application of the precision error estimate of the drag coefficient to real-world wind tunnel data.

\section{Wind Tunnel Test Results}

Data from a recent wind tunnel test of the Ames Check Standard Model (ACSM) is processed in this section to demonstrate the application of the precision error estimate of the drag coefficient to real-world test data. Figure 6 below shows the general layout of the ACSM. The ACSM is the model of a generic commercial transport type aircraft. It has a wing span of $6.5[\mathrm{ft}]$ or $1.98[\mathrm{~m}]$, a fuselage length of $7.0[\mathrm{ft}]$

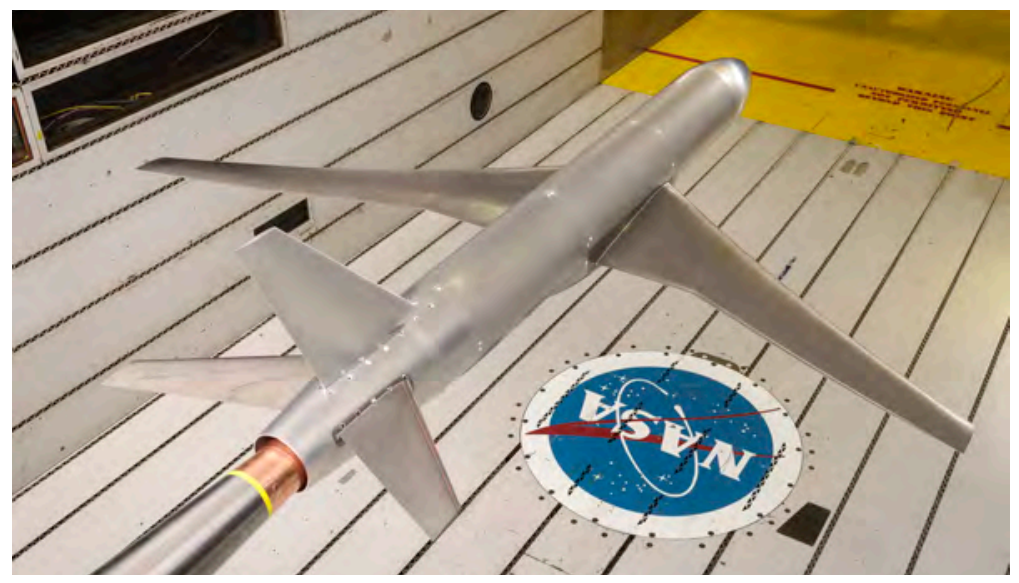

Fig. 6 Installation of the Ames Check Standard Model in the NASA Ames 11-ft Transonic Wind Tunnel.

or $2.13[\mathrm{~m}]$, and a reference area of $4.8024[\mathrm{sqft}]$ or $0.4462[\mathrm{sqm}]$. During the test the ACSM was fitted to NASA's MK34A six-component force balance. This TASK balance has a diameter of $2.5[\mathrm{in}]$ or $66[\mathrm{~mm}]$ 
and a total length of $17.3[\mathrm{in}]$ or $440[\mathrm{~mm}]$. Table 4 below shows the axial and forward/aft normal force capacities of the MK34A as only those three load components have an impact on the calculation of the precision error estimate of the drag coefficient.

Table 4: Axial and normal force capacities of the MK34A balance.

\begin{tabular}{|c|c|c|c|}
\hline & $A F$ & $N 1$ & $N 2$ \\
\hline \hline Capacity & $400[l b f]$ & $3500[l b f]$ & $3500[l b f]$ \\
\hline
\end{tabular}

Machine calibration data of the MK34A balance was analyzed and the resulting data reduction matrix for the balance load prediction was generated by using NASA's BALFIT software package (Ref. [4]). Then, precision error estimates of the drag coefficient in "counts" were computed by applying Eqs. (1a) and (1b) above. The following three inputs were used for the precision error calculation: (i) the reference area of the ACSM, (ii) the dynamic pressure at the model location in the test section, and (iii) the data reduction matrix coefficients of the balance. Consequently, the following explicit equation of the theoretical estimate of the precision error of the drag coefficient in "counts" was obtained:

$$
\left.\delta c_{D}\right|_{\text {counts }} \leq 10^{4} \cdot|\varphi| \cdot \frac{S\left(A F, R_{1}, \ldots, R_{n}\right) \cdot \cos |\alpha|+S\left(N F, R_{1}, \ldots, R_{n}\right) \cdot \sin |\alpha|}{Q \cdot A}
$$

where

$$
\begin{aligned}
& \text { wind tunnel model reference area } \Longrightarrow A=4.8024 \text { [sqft] } \\
& \text { electrical output variation bound } \Longrightarrow|\varphi|=1.0[\text { microV } / V] \\
& E q .(4) \Longrightarrow S\left(A F, R_{1}, \ldots\right) \leq \underbrace{\sqrt{\sum_{j=1}^{n}\left[\frac{\partial A F}{\partial R_{j}}\right]^{2}}}_{\text {from data reduction matrix }}=0.2691[\mathrm{lbf} /(\text { microV } / \mathrm{V})] \\
& E q .(5 b) \Longrightarrow S\left(N F, R_{1}, \ldots\right) \leq \underbrace{\sqrt{\sum_{j=1}^{n}\left[\frac{\partial N 1}{\partial R_{j}}\right]^{2}+\ldots}}_{\text {from data reduction matrix }}=4.7024[\mathrm{lbf} /(\text { microV } / \mathrm{V})]
\end{aligned}
$$

It must be pointed out that the estimate of the precision error of the drag coefficient is also a function of the angle of attack that the model experiences during the wind tunnel test (see Eq. ( $7 a)$ above). In addition, the dynamic pressure in compressible flow can be expressed as a function of (i) the Mach number and (ii) the total pressure (see Eq. (6a) or Fig. 1). Then, assuming for illustrative purposes only that the angle of attack of the model is kept at a constant value of $0.0[\mathrm{deg}]$, a contour plot of the precision error of the drag coefficient can be generated. This contour plot is shown in Fig. 7 below. 


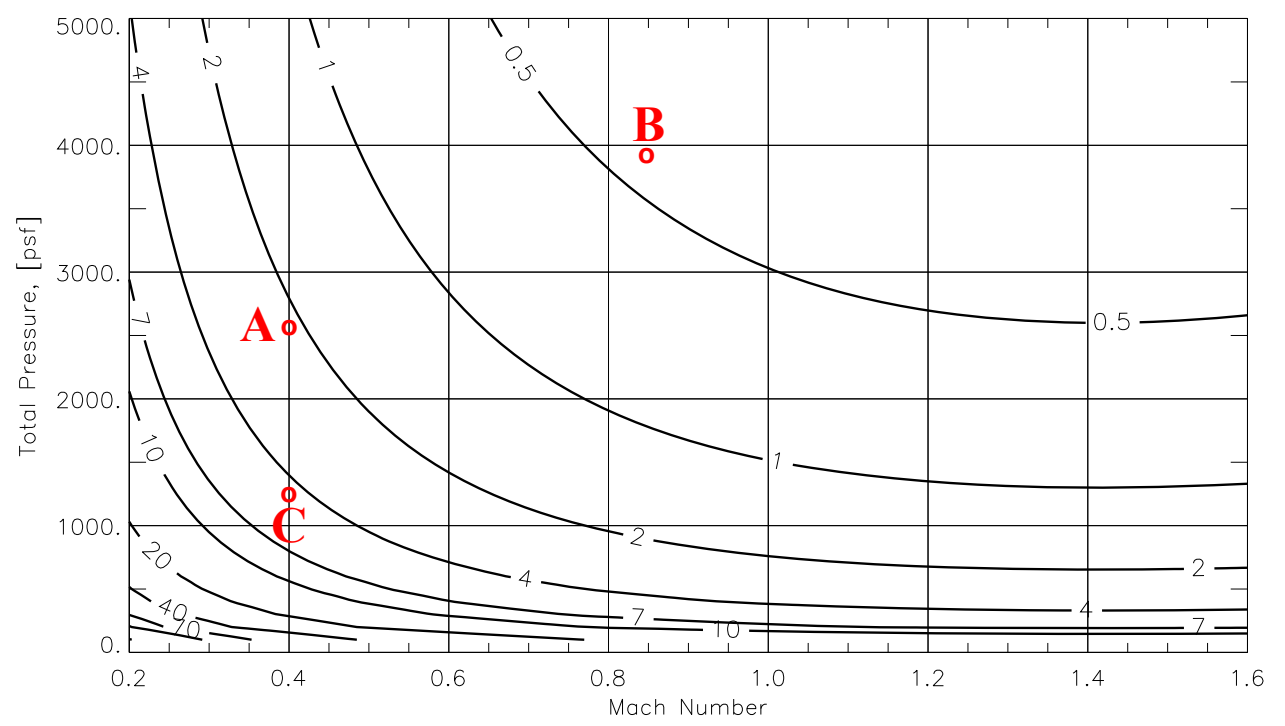

Fig. 7 Precision error of the drag coefficient plotted in "counts" versus Mach number and total pressure (Ames Check Standard Model ; MK34A ; one "count" $=0.0001$; angle of attack $=$ const. $=0^{\circ}$ ).

Now, the reader may ask the following question: how can the estimate of the precision error of the drag coefficient best be used during a wind tunnel test? First, the contour plot of the precision error above allows a wind tunnel user to quickly understand how the combination of the chosen Mach number and total pressure influences the estimated precision error of the drag coefficient. In addition, the "repeatability" of a set of wind tunnel runs is a data quality metric. It can be assessed, for example, by (i) plotting the lift coefficient versus the drag coefficient for a series of repeat runs, (ii) computing the mean of those curves, and (iii) adding/subtracting the estimate of the precision error of the drag coefficient to this mean. Ideally, data of all repeat runs should be within the region that is obtained by adding/subtracting the precision error estimate to the mean of the curves as, by design, the estimate is a conservative value. Consequently, any run located outside of this region is an "outlier". It should be investigated in more detail as it does not meet expected data quality requirements.

The authors compared sets of repeat runs at three Mach number and total pressure combinations with the precision error estimates of the drag coefficient that are shown in Fig. 7 (the three combinations are identified by using the letters $\mathbf{A}, \mathbf{B}$, and $\mathbf{C}$ ). Figure 8a below shows, for example, the lift coefficient plotted versus the drag coefficient for repeat runs 130, 132, and 135 of test condition $\mathbf{A}$. It is observed that all three

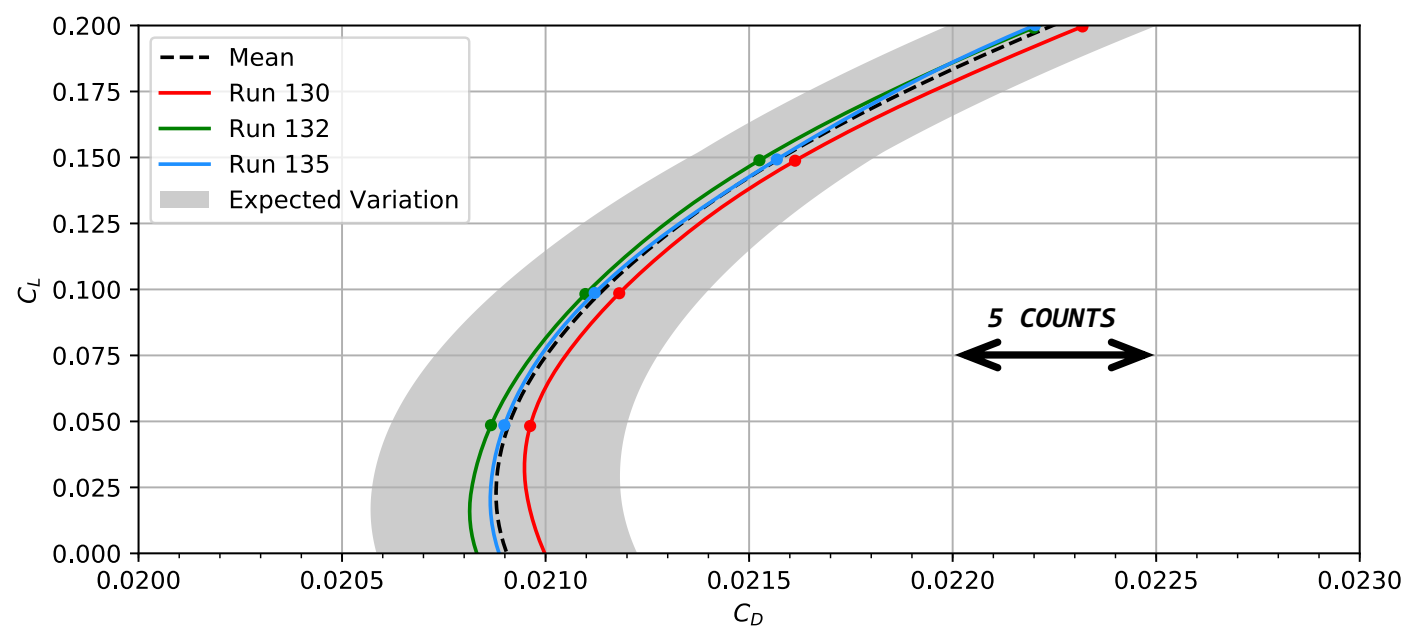

Fig. 8a Lift coefficient versus drag coefficient of the ACSM for the repeat run set 130, 132, and 135 (tunnel/test condition A in Fig. $7 \Longrightarrow M=0.40 ; P_{T}=2550[p s f] ; R e=3.0\left[10^{6} / f t\right]$ ). 
runs are within the gray region that is defined by adding/subtracting the precision error estimate of the drag coefficient to the mean of the three curves. Therefore, it is concluded that the runs meet the authors' data quality expectations. Similarly, Figure $8 \mathrm{~b}$ below shows the lift coefficient plotted versus the drag coefficient for the repeat run set 163, 165, and 166 of test condition B. Again, all runs are within the gray region that is defined by the mean of the three curves and the estimated precision error of the drag coefficient. and the authors' data quality expectations are met. The dynamic pressure of test condition $\mathbf{B}$ was 1225 [psf]. This

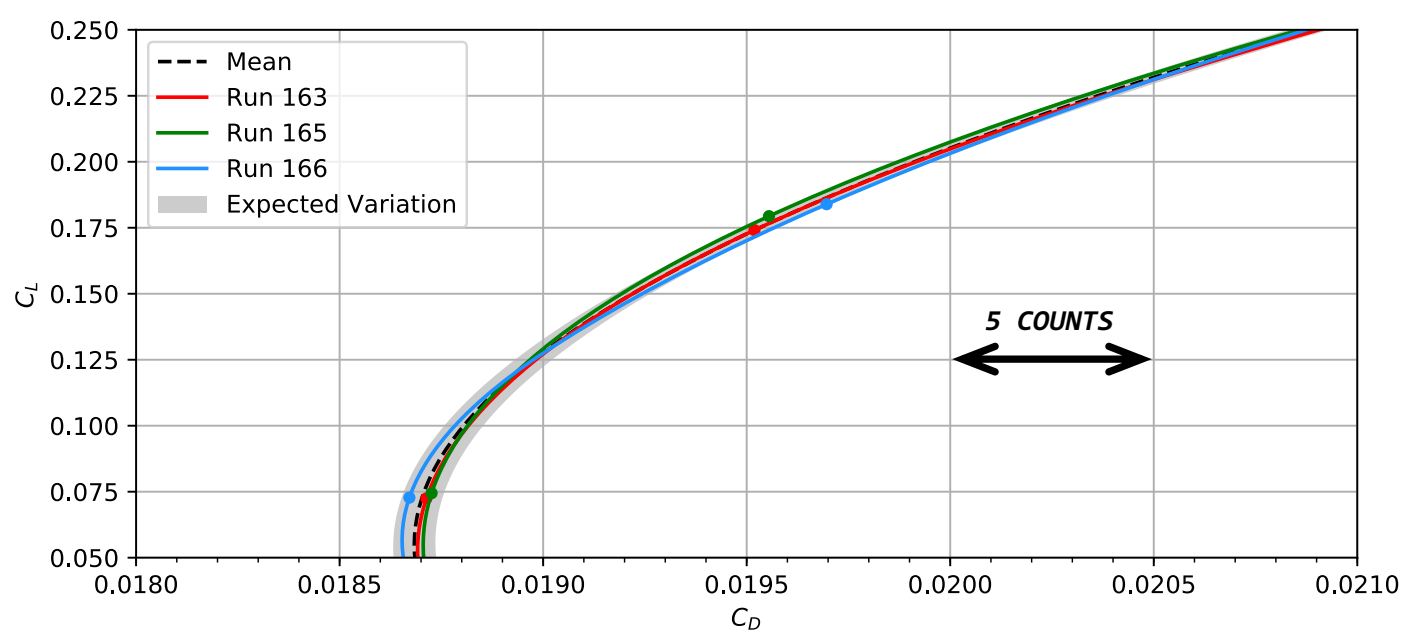

Fig. 8b Lift coefficient versus drag coefficient of the ACSM for the repeat run set 163, 165, and 166 (test condition B in Fig. $7 \Longrightarrow M=0.85 ; P_{T}=3885[p s f] ; R e=8.0\left[10^{6} / f t\right]$ ).

value is significantly larger than the dynamic pressure of $256[\mathrm{psf}]$ that the model experienced at test condition A. The difference explains the fact that the precision error of test condition $\mathbf{B}$ is substantially smaller than the precision error of test condition $\mathbf{A}$ as the dynamic pressure appears in the denominator of Eq. (7a). This conclusion is confirmed by the observation that the horizontal "width" of the gray region in Fig. 8b is much smaller than the "width" that is observed in Fig. 8a. The "width" is defined, for example, at an angle of attack of $0^{\circ}$ as twice the estimate of the error that is reported in Fig. 7. In other words, it equals $1=2 \times 0.5$ "count" for test condition B and $4=2 \times 2$ "counts" for test condition A.

Figure 9a below shows the lift coefficient plotted versus the drag coefficient for the repeat run set 117, 119 , and 124 of test condition $\mathbf{C}$. This time, it is observed that two of the three runs are outside the region

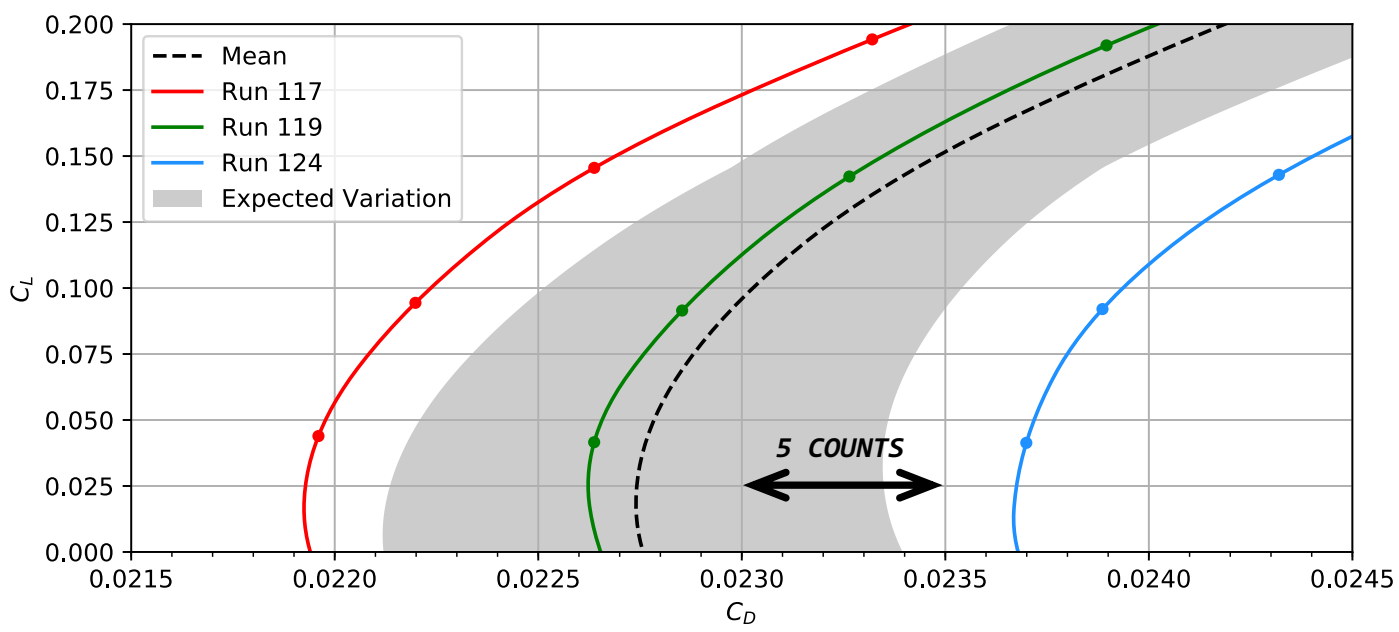

Fig. 9a Lift coefficient versus drag coefficient of the ACSM for repeat run set 117, 119, and 124 (test condition $\mathbf{C}$ in Fig. $7 \Longrightarrow M=0.40 ; P_{T}=1230[p s f] ; R e=1.5\left[10^{6} / f t\right]$ ). 
that is defined by the mean of the three curves and the estimated precision error of the drag coefficient. Therefore, the repeat runs do not meet the authors' expectations as far as data quality is concerned.

Potential sources were investigated that may have contributed to the poor repeatability of the three runs at test condition C. In those cases, the total pressure in the wind tunnel was very low (1230 [psf]). Therefore, it was suspected that hidden balance temperature changes could have negatively influenced the balance load prediction. Figure $9 \mathrm{~b}$ below shows the measured balance temperature as a function of the run

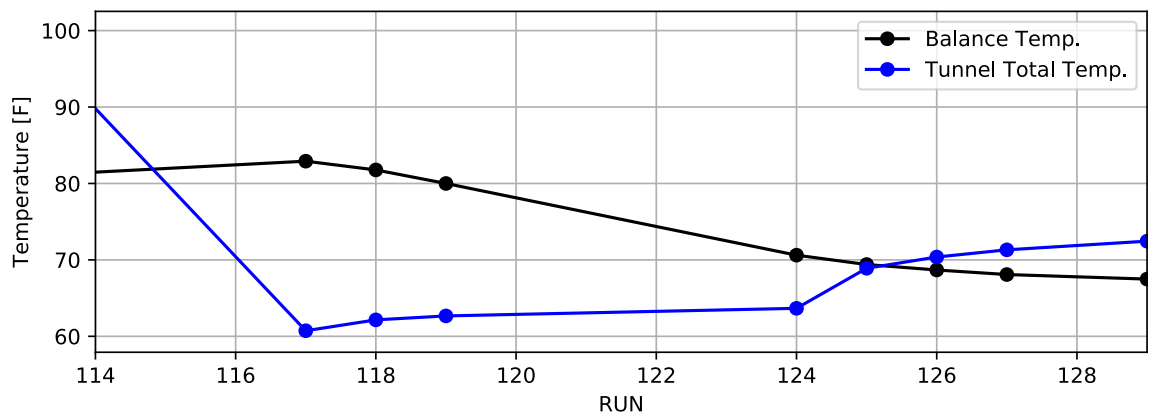

Fig. 9b Tunnel and balance temperature plotted versus the run number.

numbers. It is observed that a noticeable temperature change of about $10[\operatorname{deg} F]$ occurred after run 119. This temperature change may have introduced a hidden bias error in the output measurements of the balance that may have caused an unwanted shift in the balance load predictions for run 124 .

\section{Summary}

A new method was developed at NASA Ames Research Center that calculates a pre-test estimate of the upper bound of the precision error of a wind tunnel model's drag coefficient. Recent improvements and the application of the method to typical wind tunnel test situations were discussed in great detail. The pre-test estimate of the drag coefficient is obtained by applying a simple explicit equation that uses (i) the model reference area, (ii) the physical characteristics of the selected strain-gage balance, and (iii) the angle of attack measurement error hidden in small but observable balance bridge/gage output variations as input. This significant simplification was possible because (i) the uncertainty of the dynamic pressure measurement is small in a typical production wind tunnel facility and (ii) balance load prediction errors contained in regression models of the balance calibration data itself can be neglected as differences between drag coefficients of sets of repeat points are investigated.

In principle, the calculation of the upper bound of the precision error of the drag coefficient is defined by an explicit equation that requires the following information: the model reference area, the dynamic pressure, the angle of attack, the partial derivatives of the axial and normal forces of the balance with respect to the measured balance bridge/gage outputs, and an empirical limit of the expected balance bridge/gage output variation that is associated with the observable precision error of the angle of attack measurement. A user of the Iterative Method, for example, would directly obtain the partial derivatives of the axial and normal force of the balance from the coefficients of the linear terms of the data reduction matrix of the balance (see Ref. [2] for a description of the Iterative Method). Results of the error assessment at a constant angle of attack may be reported, for example, by generating a contour plot that depicts the precision error expressed in drag "counts" as a function of the Mach number and the total pressure. Analysis results for the combination of a generic wind tunnel model with a force balance are discussed using three different hypothetical test scenarios in order to demonstrate important characteristics of the method. Finally, experimental data from a recent wind tunnel test of the Ames Check Standard Model in the NASA Ames 11-ft Transonic Wind Tunnel was used to illustrate the application of the precision error estimates to real-world test data.

The calculation of the estimate of the precision error of the drag coefficient showed that the impact of (i) balance characteristics and (ii) the tunnel condition choice on the precision error must not be underestimated. In other words, highly accurate angle of attack measurements do not necessarily guarantee that the observed repeatability of the drag coefficient will meet expectations if a customer chooses (i) a balance 
for the wind tunnel test that has relatively low axial and normal force sensitivities and/or (ii) the model is tested at relatively low dynamic pressures.

\section{Acknowledgements}

The authors want to thank Bob Gisler, Jon Bader, and Max Amaya of NASA and Tom Volden of Jacobs Technology for their many suggestions and comments that have greatly improved the overall quality of the final manuscript of the paper. The work reported in the paper was supported by the Wind Tunnel Division at NASA Ames Research Center under contract NNA16BD26C.

\section{References}

[1] Ulbrich, N., and L'Esperance, A., "Pre-Test Assessment of the Upper Bound of the Drag Coefficient Repeatability of a Wind Tunnel Model," AIAA 2017-0484, paper presented at the 55th AIAA Aerospace Sciences Meeting, Grapevine, Texas, January 2017.

[2] AIAA/GTTC Internal Balance Technology Working Group, Recommended Practice, Calibration and Use of Internal Strain-Gage Balances with Application to Wind Tunnel Testing, AIAA R-091-2003, American Institute of Aeronautics and Astronautics, Reston, Virginia, 2003; pp. 4-5, pp. 18-19, p. 62.

[3] Ames Research Staff, Equations, Tables, and Charts for Compressible Flow, NACA Report 1135, Ames Aeronautical Laboratory, Moffett Field, California, 1953.

[4] Ulbrich, N., and Volden, T., BALFIT - Software Tool for the Regression Analysis of Multivariate Data, User Guide (revised and corrected 4th edition), Jacobs Technology Inc., prepared for NASA Ames Research Center under contract NNA09DB39C, February 2016.

[5] Ulbrich, N., and Bader, J., "Analysis of Sting Balance Calibration Data Using Optimized Regression Models," AIAA 2009-5372, paper presented at the 45th AIAA/ASME/SAE/ASEE Joint Propulsion Conference, Denver, Colorado, August 2009; p. 21, Eq. (A.23a), Eq. (A.24a). 


\section{Appendix: Upper Bound of the Partial Derivatives of the Normal Force}

\section{A. General Remarks}

The authors developed a method in 2016 that determines a pre-test estimate of the precision error of the drag coefficient of a wind tunnel model(see Ref. [1] for more details). This method uses an upper bound of the partial derivatives of the normal force of the balance with respect to the gage outputs as input. Unfortunately, the calculation of this upper bound is complicated because balance loads can also be expressed in force balance format $(A F, N 1, N 2, \ldots)$ or moment balance format $(A F, P M 1, P M 2, \ldots)$. Therefore, the authors initially used approximations of the upper bound of the partial derivatives of the normal force as input during the calculation of the precision error of the drag coefficient whenever balance data was given in either force balance or moment balance format (see Ref. [1], Eqs. (16a), 16b). There approximations can be summarized as follows:

$$
\begin{aligned}
& S\left(N F, R_{1}, \ldots, R_{n}\right) \approx S\left(N 1, R_{1}, \ldots, R_{n}\right)+S\left(N 2, R_{1}, \ldots, R_{n}\right) \\
& S\left(N F, R_{1}, \ldots, R_{n}\right) \approx \frac{S\left(P M 1, R_{1}, \ldots, R_{n}\right)+S\left(P M 2, R_{1}, \ldots, R_{n}\right)}{d}
\end{aligned}
$$

The approximations given on the right-hand sides of the above equations were recently replaced by corresponding exact expressions so that the upper bound computed in either force balance or moment balance format would match the value that is obtained if balance loads are processed in direct-read format. These two exact expressions are derived in great detail in this appendix.

The derivation of the exact expressions of the upper bound of the partial derivatives of the normal force for force balance and moment balance format starts with the original definition of the upper bound $S\left(N F, R_{1}, \ldots, R_{n}\right)$ that is given in the body of the text. Then, simply copying Eq. (5a), we can write

$$
S\left(N F, R_{1}, \ldots, R_{n}\right) \leq \sqrt{\sum_{j=1}^{n}\left[\frac{\partial N F}{\partial R_{j}}\right]^{2}}
$$

The above equation is valid for balance data that is given in direct-read format $(A F, N F, P M, \ldots)$. An exact expression of the upper bound in an alternate load format can be obtained by simply replacing the normal force on the right-hand side of Eq. (9) by the corresponding transformation that maps loads from the alternate load format to direct-read format. The derivation of the upper bound for balance loads given in force balance format is discussed in the next section of the appendix.

\section{B. Force Balance Format}

In general, the total normal force of a force balance can be computed from the predicted forward and aft normal force components by using the following transformation equation (see Ref. [5], p. 21, Eq. (A.23a)):

$$
\text { force balance format } \Longrightarrow N F=N 1+N 2
$$

Then, after replacing the total normal force given in Eq. (9) by the right-hand side of Eq. (10), we get:

$$
S\left(N F, R_{1}, \ldots, R_{n}\right) \leq \sqrt{\sum_{j=1}^{n}\left[\frac{\partial N F}{\partial R_{j}}\right]^{2}}=\sqrt{\sum_{j=1}^{n}\left[\frac{\partial(N 1+N 2)}{\partial R_{j}}\right]^{2}}
$$

It is known that the derivative of the sum of two functions equals the sum of the derivatives of the functions. Then, after applying this rule to Eq. (11a) above, we get:

$$
S\left(N F, R_{1}, \ldots, R_{n}\right) \leq \sqrt{\sum_{j=1}^{n}\left[\frac{\partial(N 1+N 2)}{\partial R_{j}}\right]^{2}}=\sqrt{\sum_{j=1}^{n}\left[\frac{\partial N 1}{\partial R_{j}}+\frac{\partial N 2}{\partial R_{j}}\right]^{2}}
$$


We also know from algebra that the following relationship is valid:

$$
\left[\frac{\partial N 1}{\partial R_{j}}+\frac{\partial N 2}{\partial R_{j}}\right]^{2}=\left[\frac{\partial N 1}{\partial R_{j}}\right]^{2}+\left[\frac{\partial N 2}{\partial R_{j}}\right]^{2}+2 \cdot\left[\frac{\partial N 1}{\partial R_{j}} \cdot \frac{\partial N 2}{\partial R_{j}}\right]
$$

Finally, after replacing the squared sum of the partial derivatives on the right-hand side of Eq. (11b) by the right-hand side of Eq. (12) and splitting the result into three separate sums, we get the exact equation of the upper bound of the partial derivatives of the normal force in force balance format:

$$
S\left(N F, R_{1}, \ldots, R_{n}\right) \leq \sqrt{\sum_{j=1}^{n}\left[\frac{\partial N 1}{\partial R_{j}}\right]^{2}+\sum_{j=1}^{n}\left[\frac{\partial N 2}{\partial R_{j}}\right]^{2}+2 \cdot \sum_{j=1}^{n}\left[\frac{\partial N 1}{\partial R_{j}} \cdot \frac{\partial N 2}{\partial R_{j}}\right]}
$$

The partial derivatives $\partial N 1 / \partial R_{j}$ and $\partial N 2 / \partial R_{j}$ listed in Eq. (13) above are easily obtained. They can be found among the coefficients of inverse matrix $\mathbf{C}_{\mathbf{1}}^{-1}$. This matrix is a part of the data reduction matrix of the given force balance assuming that the Iterative Method is used for the load prediction (for more detail see Ref. [1], p. 15, or, Ref. [2], pp. 18-19).

The exact expression of the upper bound of the partial derivatives of the normal force for loads given in moment balance format is derived in the next section of the appendix.

\section{Moment Balance Format}

It is assumed that the balance moment center of a moment balance is located halfway between the forward and aft pitching moment gages. Then, the total normal force of the moment balance can be computed from the predicted forward and aft pitching moment components by using the following transformation equation (see Ref. [5], p. 21, Eq. $(A .24 a))$...

$$
\text { moment balance format } \Longrightarrow N F=\frac{P M 2-P M 1}{d}
$$

where $P M 1$ is the forward pitching moment, $P M 2$ is the aft pitching moment, and $d$ is the distance between the pitching moment gages of the balance. Then, after replacing the total normal force in Eq. (9) above by the right-hand side of Eq. (14) and after simplifying the result, we get:

$$
S\left(N F, R_{1}, \ldots, R_{n}\right) \leq \sqrt{\sum_{j=1}^{n}\left[\frac{\partial N F}{\partial R_{j}}\right]^{2}}=\frac{1}{d} \cdot \sqrt{\sum_{j=1}^{n}\left[\frac{\partial(P M 2-P M 1)}{\partial R_{j}}\right]^{2}}
$$

It is known that the derivative of the sum of two functions equals the sum of the derivatives of the functions. Then, after applying this rule to Eq. (15a) above, we get:

$$
S(N F, \ldots) \leq \frac{1}{d} \cdot \sqrt{\sum_{j=1}^{n}\left[\frac{\partial(P M 2-P M 1)}{\partial R_{j}}\right]^{2}}=\frac{1}{d} \cdot \sqrt{\sum_{j=1}^{n}\left[\frac{\partial P M 2}{\partial R_{j}}-\frac{\partial P M 1}{\partial R_{j}}\right]^{2}}
$$

We also know from algebra that the following relationship is valid:

$$
\left[\frac{\partial P M 2}{\partial R_{j}}-\frac{\partial P M 1}{\partial R_{j}}\right]^{2}=\left[\frac{\partial P M 1}{\partial R_{j}}\right]^{2}+\left[\frac{\partial P M 2}{\partial R_{j}}\right]^{2}-2 \cdot\left[\frac{\partial P M 1}{\partial R_{j}} \cdot \frac{\partial P M 2}{\partial R_{j}}\right]
$$

Finally, after replacing the squared sum of the partial derivatives on the right-hand side of Eq. (15b) by the right-hand side of Eq. (16) and splitting the sum into three separate sums, we get the exact equation of the upper bound of the partial derivatives of the normal force in moment balance format:

$$
S\left(N F, R_{1}, \ldots, R_{n}\right) \leq \frac{1}{d} \cdot \sqrt{\sum_{j=1}^{n}\left[\frac{\partial P M 1}{\partial R_{j}}\right]^{2}+\sum_{j=1}^{n}\left[\frac{\partial P M 2}{\partial R_{j}}\right]^{2}-2 \cdot \sum_{j=1}^{n}\left[\frac{\partial P M 1}{\partial R_{j}} \cdot \frac{\partial P M 2}{\partial R_{j}}\right]}
$$

The partial derivatives $\partial P M 1 / \partial R_{j}$ and $\partial P M 2 / \partial R_{j}$ listed in Eq. (17) above are easily obtained. They can be found among the coefficients of inverse matrix $\mathbf{C}_{\mathbf{1}}^{-1}$. This matrix is a part of the data reduction matrix of the given moment balance assuming that the Iterative Method is used for the load prediction (for more detail see Ref. [1], p. 15, or, Ref. [2], pp. 18-19). 